\title{
Reflets
}

Revue ontaroise d'intervention sociale et communautaire

\section{La pratique de la physiothérapie en milieu minoritaire : mon expérience en tant que francophone à l'Hôpital de Hamilton}

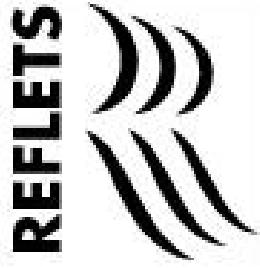

\section{Anik Goulet}

Volume 8, numéro 1, printemps 2002

La réadaptation : son visage français en Ontario

URI : https://id.erudit.org/iderudit/026381ar

DOI : https://doi.org/10.7202/026381ar

Aller au sommaire du numéro

Éditeur(s)

Reflets : Revue ontaroise d'intervention sociale et communautaire

ISSN

1203-4576 (imprimé)

1712-8498 (numérique)

Découvrir la revue

Citer cet article

Goulet, A. (2002). La pratique de la physiothérapie en milieu minoritaire : mon expérience en tant que francophone à l'Hôpital de Hamilton. Reflets, 8(1),

164-168. https://doi.org/10.7202/026381ar

Tous droits réservés (C) Reflets : Revue ontaroise d'intervention sociale et communautaire, 2002
Ce document est protégé par la loi sur le droit d'auteur. L'utilisation des services d'Érudit (y compris la reproduction) est assujettie à sa politique d'utilisation que vous pouvez consulter en ligne.

https://apropos.erudit.org/fr/usagers/politique-dutilisation/ 


\section{La pratique de la physiothérapie en milieu minoritaire : mon expérience en tant que francophone à l'Hôpital de Hamilton}

Anik Goulet

Physiothérapeute, Stoney Creek

Une physiothérapeute franco-ontarienne approche pour la première fois un client hospitalisé dans le Sud de l'Ontario. Cet homme, âgé de 72 ans, a subi une chirurgie à la hanche.

Physiothérapeute (Pht) :

— Good morning Mr. Beauregard, how are you feeling today?

Client: $(C)$ :

- (avec un accent français) Well.

Pht : - My name is Julie and I am a physiotherapist. Your Doctor asked me to come see you to help you start walking again. Do you consent to this?

C : - (accent français) Dat is fine.

D'après son accent, la physiothérapeute se doute que ce client est francophone.

Pht : - Do you speak French?

$C: \quad$ - Ben oui je parle français ma grande!

Pht : - Aimeriez-vous que je vous parle en français?

$C: \quad-$ Ben sûr que j'aimerais ça. (Le client sourit, content de recevoir des services dans sa langue maternelle) 
Ce scénario est typique d'une première rencontre entre une physiothérapeute et un client francophone (non-identifié comme tel) dans le Sud de l'Ontario. En effet, il est tout à fait normal en milieu anglophone de s'adresser en anglais à chaque client, peu importe son nom de famille. Les services en français dans le Sud de l'Ontario ne sont pas acquis d'emblée. Ils sont plutôt rares. Par ailleurs, des efforts substantiels sont entrepris afin d'enrichir les services en français.Vivre en milieu minoritaire, c'est ma réalité depuis toujours! Aujourd'hui, dans mon rôle de physiothérapeute, cette réalité m'apporte beaucoup de frustrations mais encore beaucoup plus d'espérances.

\section{Mon parcours en bref}

Je suis une Franco-Ontarienne de Hamilton, une ville majoritairement anglophone. J'ai fait mes études élémentaires et secondaires en français aux écoles Monseigneur-de-Laval et Georges-P.-Vanier.Après le secondaire, j'ai été admise à l'Université d'Ottawa, dans le programme de physiothérapie, le seul programme de physiothérapie offert en français en Ontario. À Ottawa,je fus étonnée que l'on puisse recevoir en français toutes sortes de services de qualité, et cela sans ou avec peu d'effort.

À la fin de mes études, je suis retournée dans ma ville natale pour y entamer ma nouvelle carrière. J'avais comme objectif d'offrir des services de physiothérapie dans ma langue maternelle aux 5775 francophones de Hamilton. Ce projet me semblait réaliste au départ. Ma première démarche a été de contacter le Centre de santé communautaire francophone pour offrir mes services. Toutefois, le Centre ne possédait pas les fonds nécessaires pour dispenser, aux requérants, des services directs en français dans le domaine de la physiothérapie. Ce scénario est fréquent dans les milieux minoritaires. Néanmoins, j’ai offert mes services à titre de bénévole pour des présentations ciblées, par exemple la prévention des chutes pour les personnes âgées. 
Par ailleurs, j'ai accepté un poste en médecine générale au Centre médical universitaire de McMaster. Là, je travaille avec une équipe médicale formidable, mais majoritairement anglophone. L'Hôpital McMaster est un des quatre hôpitaux du réseau de la Corporation des sciences de la santé de Hamilton. Quatorze physiothérapeutes, dont deux francophones, travaillent pour cette corporation.

Durant mes sept premiers mois de travail, j'ai eu l'occasion d'intervenir auprès de six clients francophones. Ceux-ci ont affirmé avoir bien apprécié les services en français. Toutefois, je me demande combien de clients n'ont pas pu recevoir des traitements de physiothérapie en français parce qu'ils n'ont pas eu la chance d'être référés a l'une des deux physiothérapeutes francophones. Décidément, je peux conclure qu'il y a un manque de ressources dans la région de Hamilton pour les francophones.

Le récent rapport de la FCFA (2001), parrainé par Santé Canada, confirme qu'en effet les francophones vivant en situation minoritaire présentent un niveau de santé d'environ $20 \%$ inférieur aux autres communautés majoritaires. La difficulté à obtenir des services de santé dans sa langue maternelle est sûrement un des facteurs importants. Comprendre les consignes et les informations qui nous sont données et pouvoir décrire dans sa langue son état de santé peut certainement avoir un impact sur sa propre réadaptation. Comment prendre une décision éclairée au sujet d'un traitement médical important alors qu'on ne comprend pas très bien ce qui nous est expliqué?

\section{Efforts pour améliorer les services en français à Hamilton}

Voilà qu'à Hamilton, la communauté francophone minoritaire a décidé d'améliorer son sort. En 2001, plusieurs professionnels francophones et anglophones des hôpitaux de Hamilton et des leaders de la communauté francophone se sont réunis à plusieurs 
reprises pour rédiger et proposer un plan d'action. Notons que ces réunions ont été dirigées en grande partie par des anglophones. C'est cela être minoritaire! Plusieurs stratégies adoptées concernent l'amélioration des services en français dans les salles d'urgence de Hamilton. En voici quelques-unes.

\section{1- Aux affaires publiques :}

- mettre sur pied une campagne auprès des médias locaux préconisant une approche positive afin de reconnaitre le bilinguisme dans les hôpitaux de Hamilton.

- développer des services en français et les utiliser comme modèles pour éventuellement offrir des services aux autres communautés minoritaires puisque Hamilton est une ville multiculturelle.

\section{2- Au comité des formulaires :}

- recenser les formulaires les plus souvent utilisés par les patients ou leurs familles et créer des formulaires bilingues.

\section{3- Aux ressources externes :}

- Identifier les ressources francophones de la région, comme par exemple le Centre de santé et les écoles francophones afin de former une synergie de collaboration et d'information.

Ces stratégies et plusieurs autres sont présentement en développement. Finalement, des efforts concrets! Jusqu'à présent, les progrès sont lents mais substantiels. Malgré ces progrès, les ressources francophones sont encore limitées dans le Sud de l'Ontario, surtout à Hamilton. Les groupes de pression sont confiants en l'avenir, même s'il n'y a pas beaucoup de fonds pour appuyer ce mouvement et faire avancer la cause.

\section{Frustrations}

C'est parfois frustant d'être une physiothérapeute francophone dans un milieu anglophone. C'est difficile de pratiquer et de 
sauvegarder sa langue, quand on ne s'en sert pas d'une façon régulière. De temps à autres, je me sens isolée. Le désir de faire carrière dans un milieu plus francophone, comme Ottawa, m'attire. Ce serait tellement plus facile il me semble!

Par contre, lorsque je pense aux besoins et à la survie des services en français dans ma communauté de Hamilton, je me dis qu'on a besoin de professionnels de la santé, francophones comme moi, pour maintenir et améliorer le peu de services que nous avons en français dans la région. Ma coordonnatrice des services en français du Conseil régional de santé de Hamilton me l'a répété. Elle a su m'inspirer...

\section{Des voeux}

Le désir d'aider les francophones de ma région surpasse de loin mes frustrations. Je suis certaine qu'il sera possible un jour d'offrir des services de physiothérapie de qualité en français aux gens de la communauté francophone de Hamilton. Enfin, je suis devenue physiothérapeute pour aider toutes les personnes qui sollicitent mon aide, francophones ou non. C'est mon travail en ce moment à Hamilton. Malgré les progrès et les efforts pour améliorer les services en français, il reste toujours des problèmes à résoudre et de nouveaux défis à relever pour une physiothérapeute francophone en milieu minoritaire. Pour l'instant, je reste ici avec ma famille et mes amis francophones. Je suis toujours ambassadrice de la langue française dans le Sud de l'Ontario en tant que physiothérapeute qui aime son travail.

\section{Références}

www.ofa.gov.on.ca/ofamap/French/index.html 